\title{
Research Paper \\ Comparing the Knee Joint Kinematic Parameters of Female Athletes During Sidestep Cutting Task Before and After Fatigue in Predictable and Unpredict- able Settings
}

\author{
*Elham Hosseini ${ }^{1}(1)$, Abdolhamid Daneshjoo $^{1}\left(\right.$ Mansour Sahebozamani $^{1}{ }^{(0)}$
}

1. Department of Sports Injuries and Corrective Exercises, Faculty of Sports Sciences, Shahid Bahonar University of Kerman, Kerman, Iran.

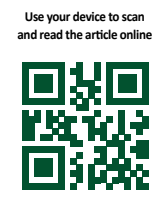

Crtation: Hosseini E, Daneshjoo A, Sahebozamani M. [Comparing the Knee Joint Kinematic Parameters of Female Athletes During Sidestep Cutting Task Before and After Fatigue in Predictable and Unpredictable Settings (Persian)]. Journal of Sport Biomechanics. 2019; 5(3):178-187. https://doi.org/10.32598/biomechanics.5.3.5

doi'https://doi.org/10.32598/biomechanics.5.3.5

Key words:

Kinematics, Knee injuries, Knee flexion, Knee valgus.

\section{ABSTRACT}

Article Info:

Received: 01 Feb 2019

Accepted: 18 Sep 2019

Available Online: 01 Dec 2019

\section{Extended Abstract}

\section{Introduction}

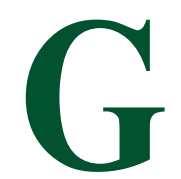

iven the increasing number of athletes in the world in a variety of sports, as well as the increasing prevalence of sports injuries, it is more important to carefully examine the conditions that athletes face during training and competitions, and can increase the risk of injury in them.
Despite advances in prevention, injuries such as Anterior Cruciate Ligament (ACL) injury and its negative consequences are still on the rise among athletes. Such injuries are non-traumatic and, therefore, kinematic parameters during exercise maneuvers, especially in unpredictable conditions and fatigue, can be considered as important risk factors and can expose athletes to ACL injury.

Women are more vulnerable than men and adolescents more than adults, and the dangerous conditions mentioned

\section{* Corresponding Author:}

Elham Hosseini

Address: Department of Sports Injuries and Corrective Exercises, Faculty of Sports Sciences, Shahid Bahonar University of Kerman, Kerman, Iran.

Tel: +98 (913) 9382891

E-mail: hosseinelham7400@sport.uk.ac.ir 
above have different effects on these sex and age groups for different reasons. Numerous studies have also shown that after ACL injury, there are countless consequences for the person and often lead to the end of the injured person's athletic career. These factors indicate the importance of further research to develop appropriate injury prevention programs at critical ages such as adolescence.

However, little research has focused on girls in this age group. Moreover, despite the fact that athletes face all these factors during training and competition, no research was found that has examined the effect of conditions such as fatigue along with the unpredictability of sports skills in these gender and age groups. Therefore, the aim of this study was to compare the kinematic parameters of the knee joint before and after functional fatigue during lateral movements (sidestep cutting) in predictable and unpredictable settings in adolescent female athletes.

\section{Methods}

The is a quasi-experimental study conducted in Laboratory of Physical Education of Sports Sciences Faculty at Shahid Bahonar University in Kerman, Iran in summer 2019. The study population consisted of all adolescent female athletes in Kerman. The sample size was determined 49 using the GPower program and considering the statistical power of 0.9 and a significance level of 0.05 . In this regard, 49 samples (mean age: $14.691 \pm 0.146$ years, mean $\mathrm{BMI}=49.058 \pm 1.226$ $\mathrm{kg} / \mathrm{m}^{2}$, mean height $=1.618 \pm 0.008$ ) were selected using purposive and convenience sampling methods. All subjects ranged in age from 13 to 16 years, and had at least 3 years of regular exercise (3 sessions per week) and a normal BMI.

Those who had a history of lower limb injury or surgery in the past 6 months, or had participated in an ACL injury prevention program or had heavy physical activities 24 hours before the testing were excluded from the study. After holding a briefing session and completing the individual forms, the assessments were performed in two different days. On the first day, clinical measurements (height and weight) were performed, and functional tests (speed, jump and agility) and knee joint kinematics measurement during sidestep cutting maneuver were carried out on the second day. In order to record the three-dimensional kinematics of subjects, markers were used on the lower limbs in three dimensions. Participants were then asked to perform a sidestep cutting movement in predictable setting (towards the dominant leg) and unpredictable setting (recognizing the direction of movement using the flashing light).

For the sidestep cutting maneuver, first the subject was asked to run the specified distance of 10 meters with her maximum speed and then make a vertical jump towards the ball that was adjusted according to her vertical jump height. After landing, she ran a distance of 3 meters again and performed sidestep cutting at the specified angle (40 degrees). Eventually, subjects reached exhaustion using the Bruce protocol, and reported their fatigue according to the Borg scale. The kinematic parameters during sidestep cutting maneuver were recorded by a 6-camera optical motion analysis system at a sampling frequency of $200 \mathrm{~Hz}$. Collected data were analyzed in MATLAB and SPSS. The Mixed Repeated Measure test was used to determine the differences in kinematic parameters between and within groups. One-way ANOVA test was used to eliminate the effect of interfering factors such as agility and speed. With non-significant difference in these factors, the homogeneity of study groups was confirmed.

\section{Results}

According to the mixed repeated measure ANOVA results, the change in kinematic factors such as knee flexion $(\mathrm{P}=0.001)$, knee valgus $(\mathrm{P}=0.001)$ and tibia rotation

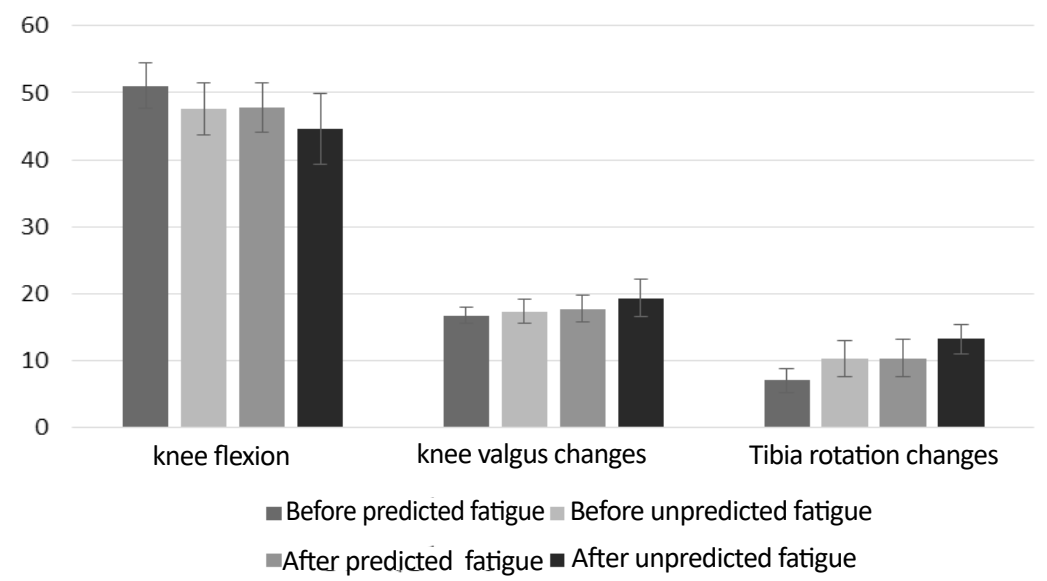

Figure 1. Changes in kinematic parameters under different fatigue conditions

Journal of Sport Biomechanics 
( $\mathrm{P}=0.001)$ before and after fatigue and also during predictable and unpredictable cutting maneuvers were significantly different (Figure 1).

\section{Discussion}

Despite little research in the field of three-dimensional kinematics, there were some studies whose were in agreement or disagreement with the present study, indicating that the kinematic parameters of the knee joint are related to each other. Most studies have shown a decrease in knee flexion, and an increase in knee valgus and tibia rotation. All studies have shown that these changes can increase the pressure on the knee joint, especially the ACL, and ultimately increase the amount of injury. Overall, it was concluded that performing unpredictable cutting maneuvers, especially in time of fatigue, is associated with a high risk of injury in adolescent female athletes. This highlights the importance of creating the right training methods, training programs and proper injury prevention.

\section{Ethical Considerations}

\section{Compliance with ethical guidelines}

All ethical principles were considered in this article. The participants were informed about the purpose of the research and its implementation sages; they were also assured about the confidentiality of their information; Moreover, They were allowed to leave the study whenever they wish, and if desired, the results of the research would be available to them. This study was approved by the Ethics Committee of Shahid Bahonar University of Kerman (Code: IR.UK.VETMED.REC.1398.022).

\section{Funding}

The present paper was extracted from the MSc. thesis of the first author, Department of Sports Injuries and Corrective Exercises, Faculty of Sports Sciences, Shahid Bahonar University of Kerman.

\section{Authors' contributions}

Conceptualization, Methodology, Supervision: All authors; Invesigation, Writing original draft, Funding acquisition, Resources: Elham Hosseini; Writing-review and editing: Abdolhamid Daneshjoo, Mansour Sahebozaman.

Conflicts of interest

The authors declared no conflict of interes.

\section{Acknowledgements}

The authors would like to thank Saeed Mollahosseini and Milad Fallahzadeh for their cooperation in the research process. 


\title{
مقايسه يار امترهاى كينماتيكى مفصل زانوقبل وبعداز خستكى حين برش جانبى باوبدون بيشبينى دور وزشكاران دختر
}

\author{
"الهام حسينى' ه، عبدالحميد دانشجو' ن، منصور صاحبالزمانى' \\ 1. كووه آسيبشناسى ورزشى و حركات اصلاحى، دانشكده تربيتبدنى و علوم ورزشى، دانشكاه شهيد باهنر، كرمان، ايران.
}

\begin{abstract}
حكيد

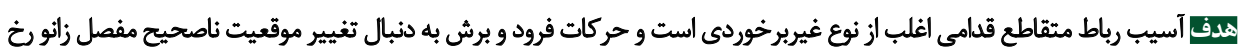

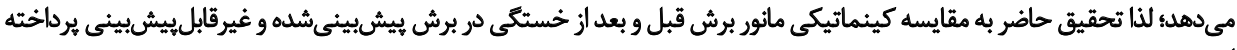
است.

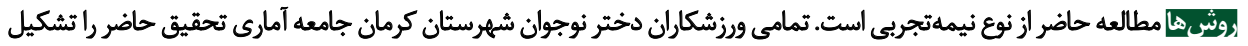

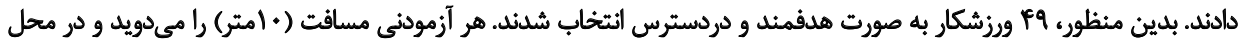

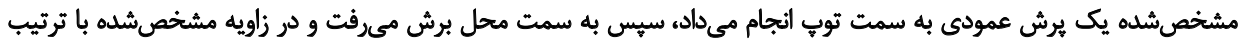

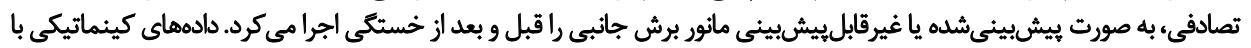

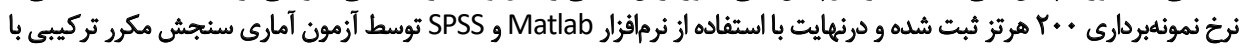

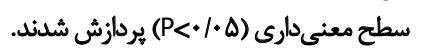

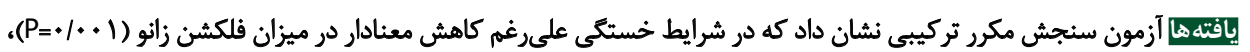

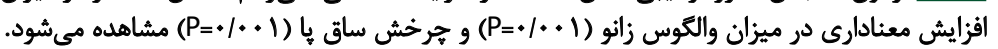

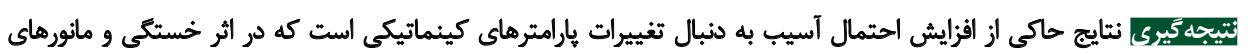

كليدواثوها:

كينماتيك، آسيب زانو، فلكشن زانو، والكوس زانو، زانو

و عملكرد حس عمقى و درنهايت اختلال در يايدارى مفصل

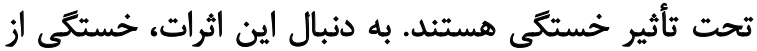

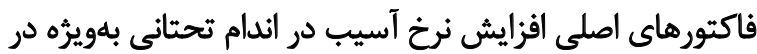

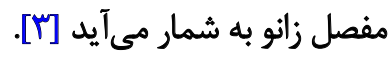

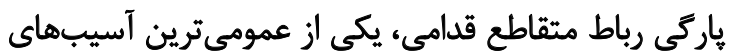

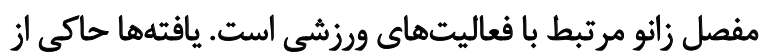

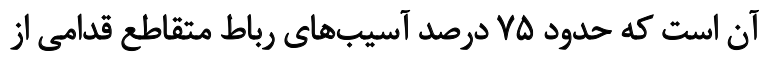

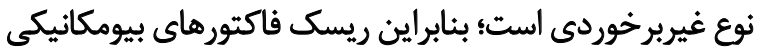

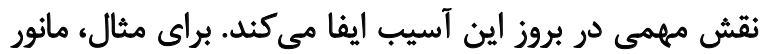

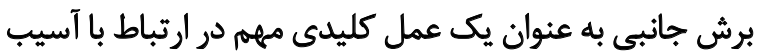

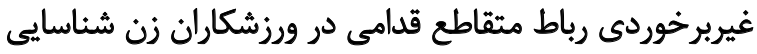

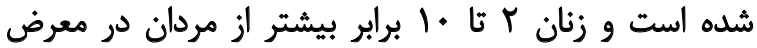

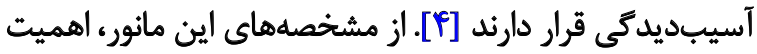

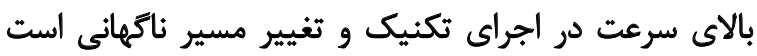

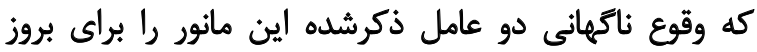

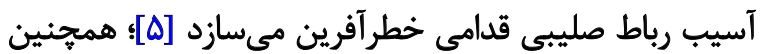

ازآنجاكه ورزشكاران در شرايط بيجيجيده و متغير بازى به طور

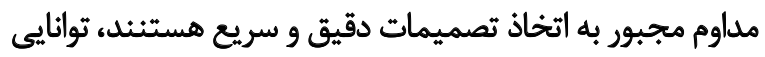

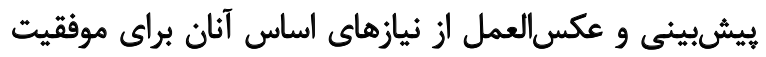

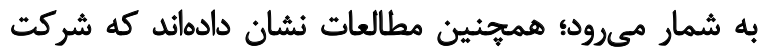

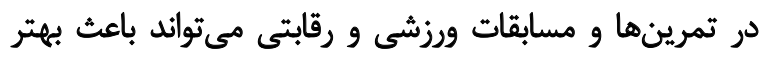

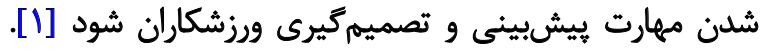

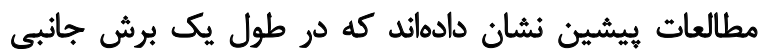

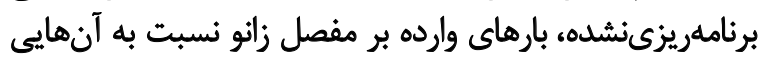

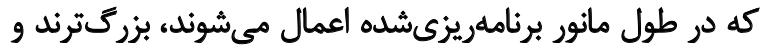
همكن است منجر به آسيب در اين ناحيه شوند [باند]. عامل ديكرى كه مي تواند عملكرد ورزشكار را تحت تأثير قرار

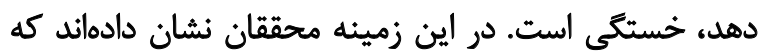

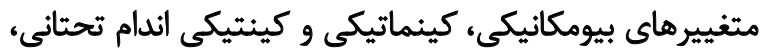

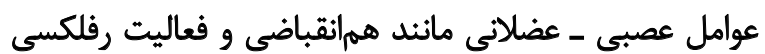

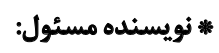

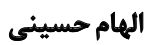
نشانى: كرمان، دانشكاه شهيد باهنر، دانشكده تربيتبدنى و علوم ورزشى، كروه آسيبشناسى ورزشى و حركات اصلاحى. تلفن: يست الكترونيكى: hosseinelham7400@sport.uk.ac.ir 
و يا Jايل داعت قبل از انجام تست فعاليت شديد بدنى داشتند، به دليل داشتن معيارهاى خروج از تحقيق خارج شدائد فيد

قبل از انجام تحقيق، اهميت، روش كار و اجراى آزمون به

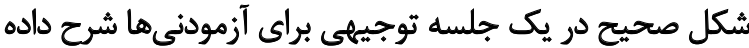

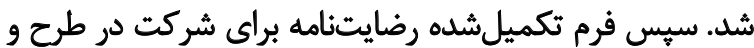

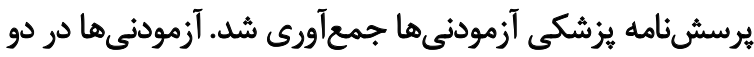

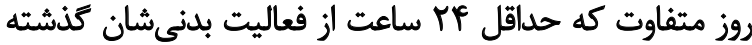

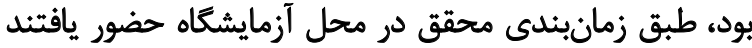

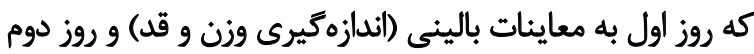

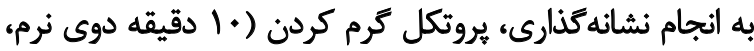
حركات كششى و قدرتى اختصاصى اندام تحتانى) و انجام يروتئي مانور برش جانبى ونمونهبردارى كينماتيكى اختصاص اختصاص داده شد. در حين انجام مراحل تست كيرى سعى شد كه شرايط

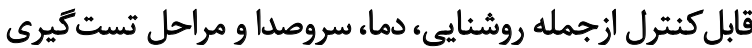

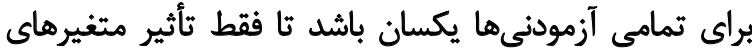

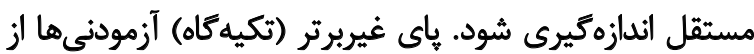

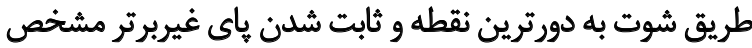

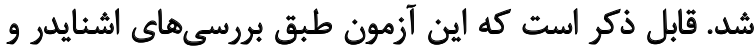

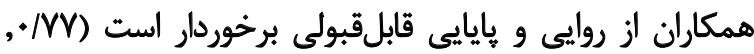
(r=-/Ar=ICC

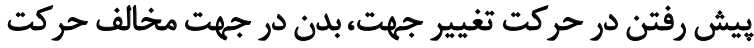

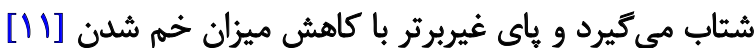

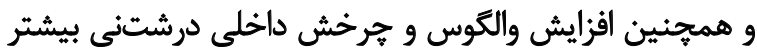

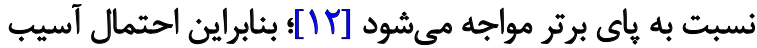

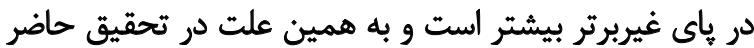

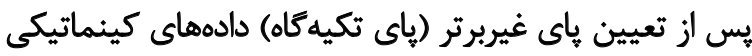

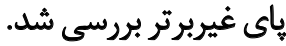

در ادامه براى ارزيابى شاخصهاي كينماتيكى فلكشن، والقوس

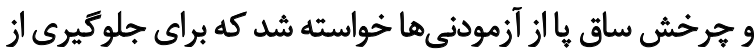

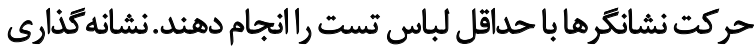

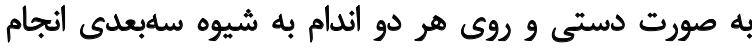

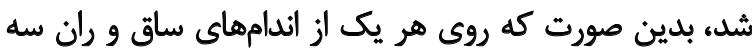

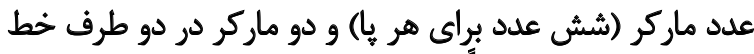

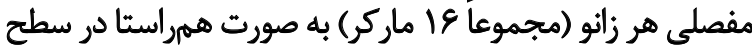

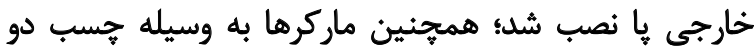

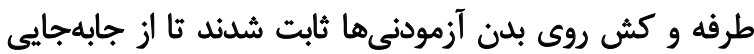

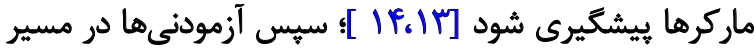

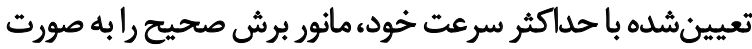

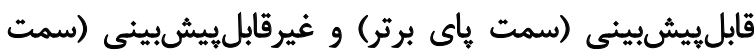

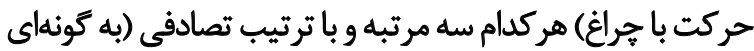

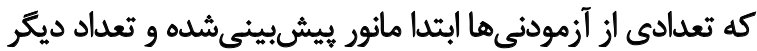

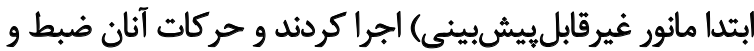

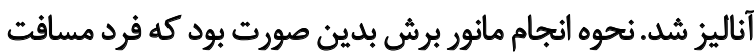

در تحقيقات ذكر شده است كه حركت برش جانبى اجراشده

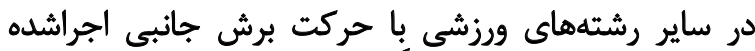

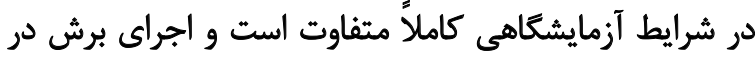

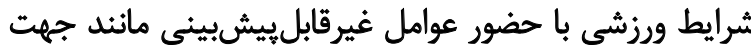

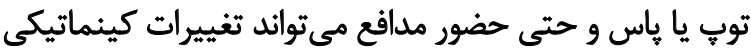

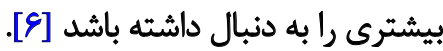

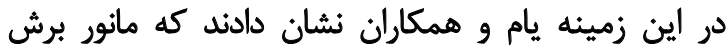

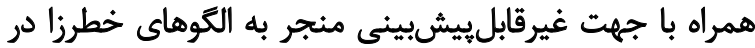

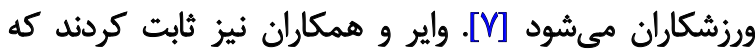

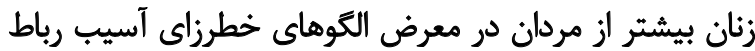

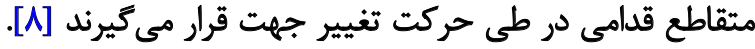

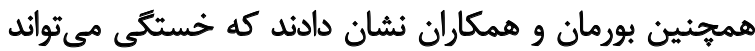

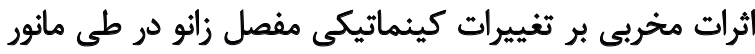

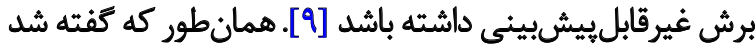

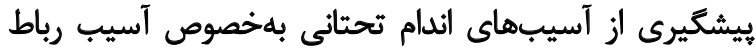

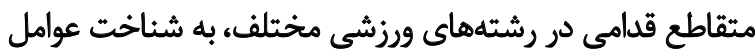

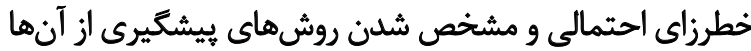

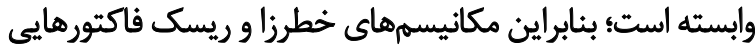

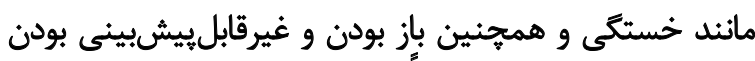

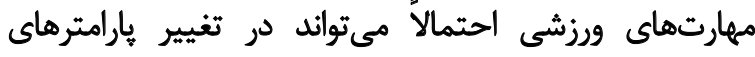

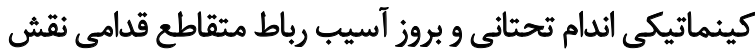

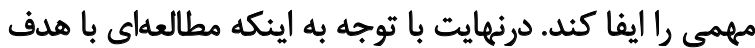

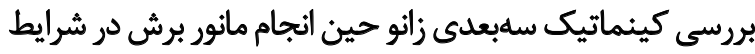

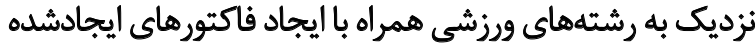

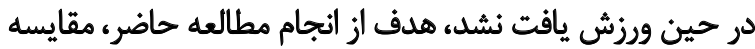

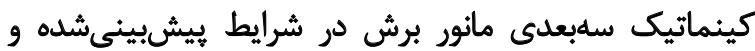

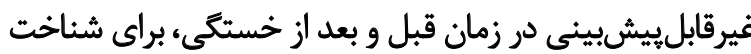
هرجه بهتر شاخصهاي كينماتيكى در وضعيتهاى خطرز

$$
\text { روششناسى }
$$

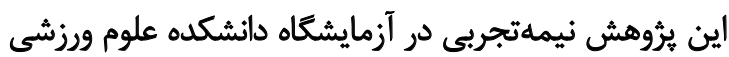

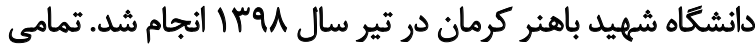

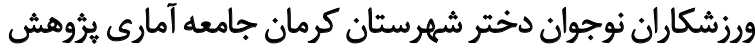

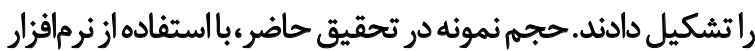

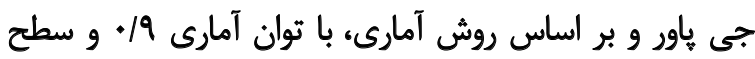

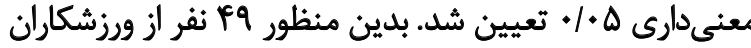

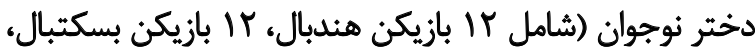

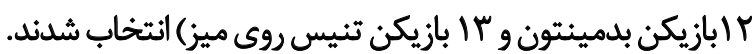

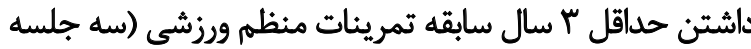

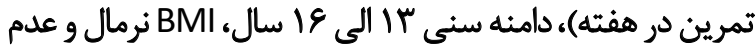

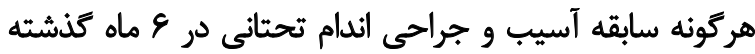

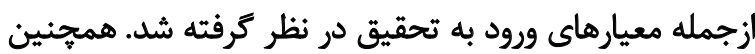

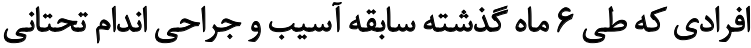

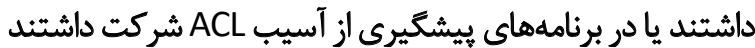




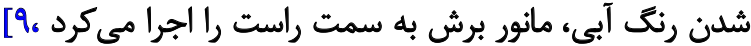

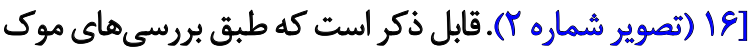

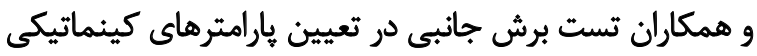

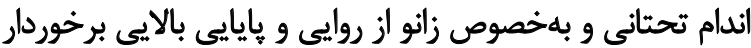

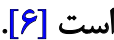

در مرحله بعد، آزمودنى با اجراي آزمون زيربيشينه بروس (مداي

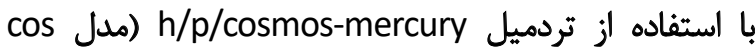

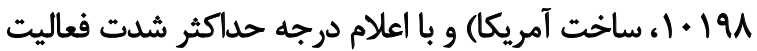

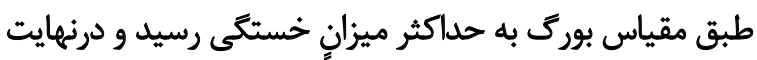

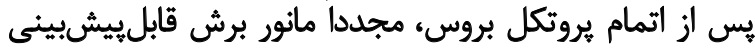

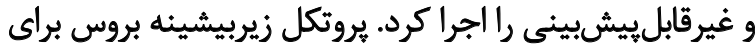

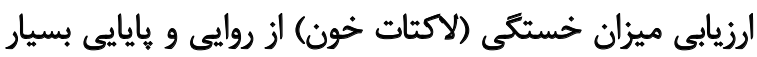

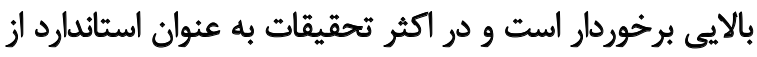

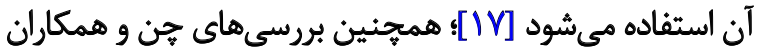

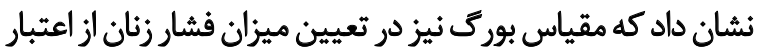

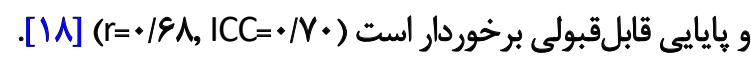

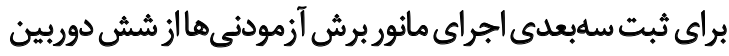

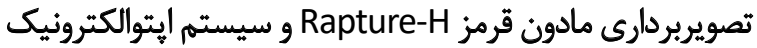
سلبعدى Motion Analysis شركت Motion Analysis

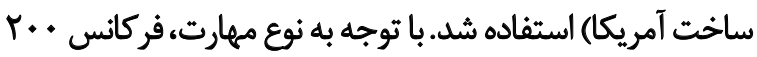

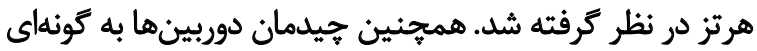

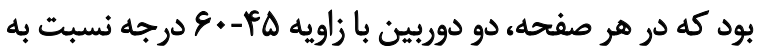

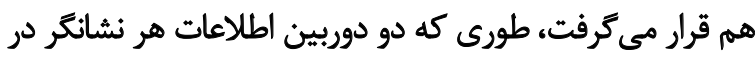

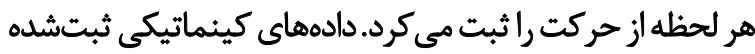

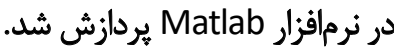

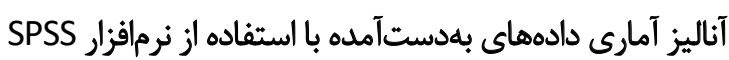

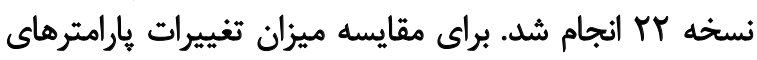

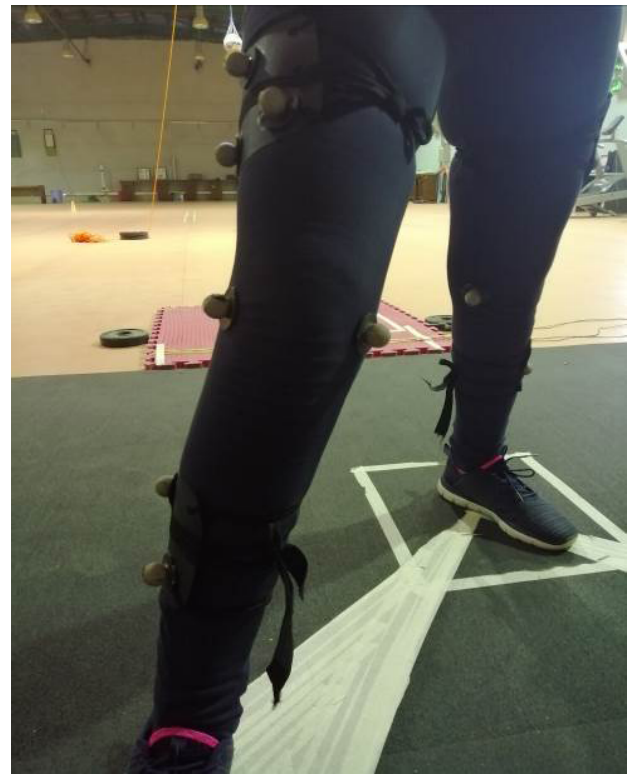

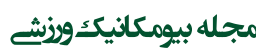

تصوير ا. لحظه تغيير جهت آزمودنىها در مانور برش جانبى بيشبينى بـده به سمت بايى برتر

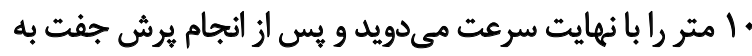

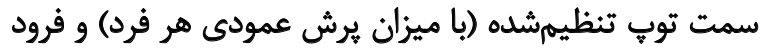

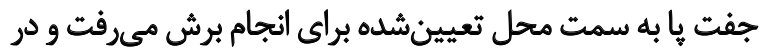

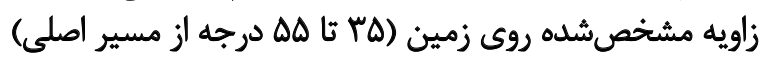

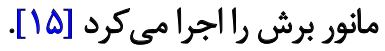

در برش قابل بيشبينى فرد به سمث پإى برتر (تصوير شماره

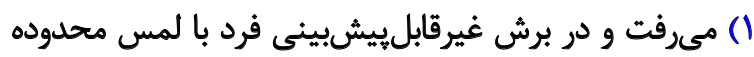

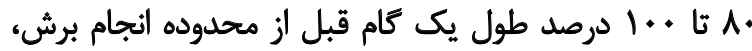

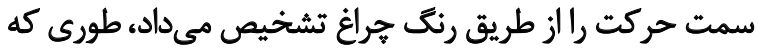

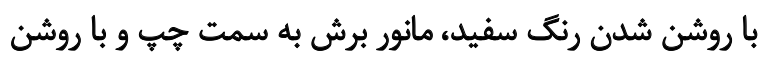

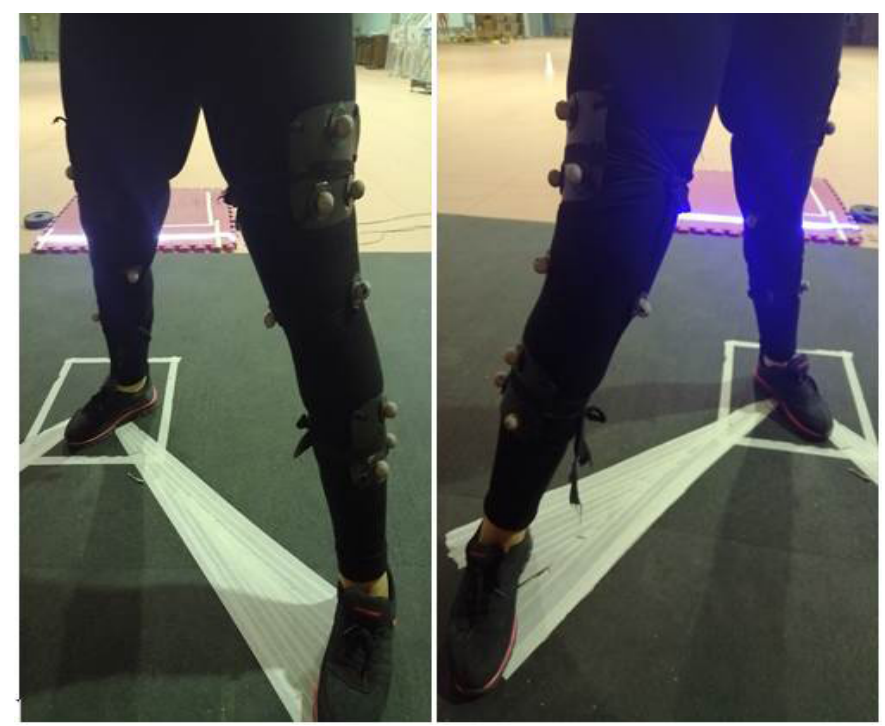

مجله بيومكانيك ورنش

تصوير r. لحظه اجراى مانور برش جانبى غيرقابل بيشبينى و تشخيص سمت حركت همراه با روشن شدن جراغ 


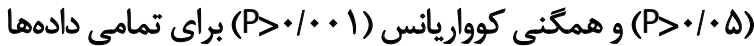

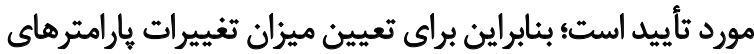

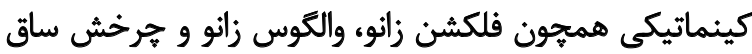

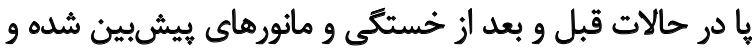

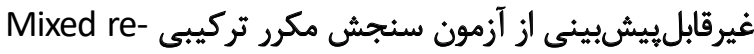
ANOVA One استفاده شد. نتايج تسكر تركيبي

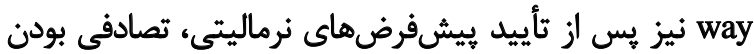

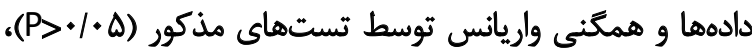

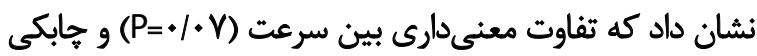
( آزمودنى ها وجود ندارد.

در جدول شماره ا نتايج آزمون Wilks lambda، ميزان

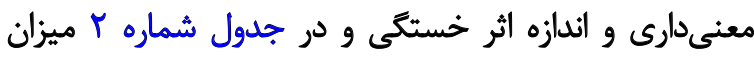

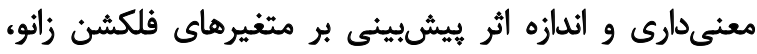

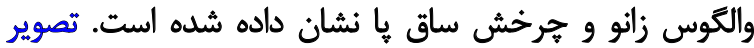

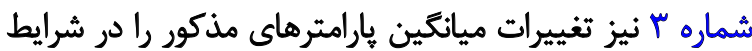

مختلف تحقيق نشان مى دهد.

بs

يُشازاين، تحقيقات فراوانى به تأثير جداكانه ريسك

كينماتيكى طى مانور برش قابل يبيشبينى و غيرقابل ييشبينى،

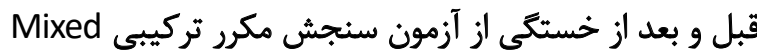
repeated measure

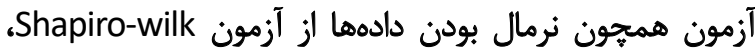

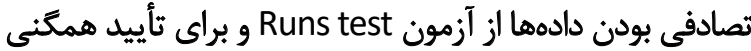

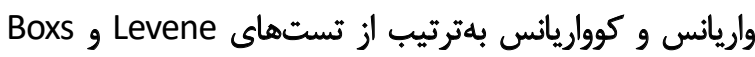

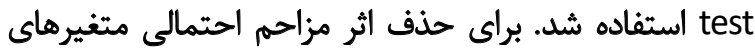

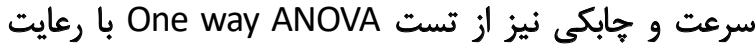

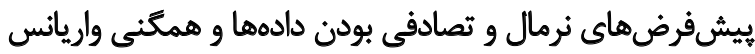

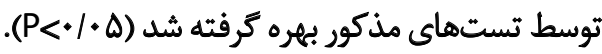

نتايج

در مطالعه حاضر Fq ورزشكار نوجوان دختر با ميانكين

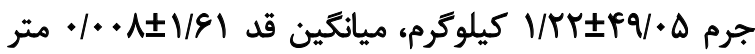

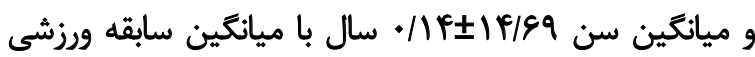

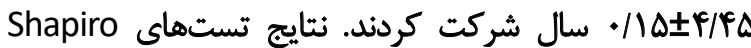
Runs test و wilk

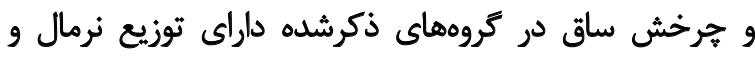

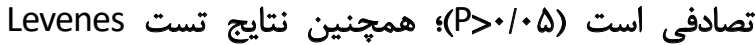

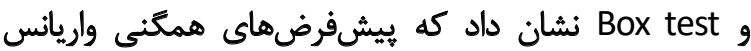

جدول ا.نتايج اثرات خستئى بر فاكتورهاى كينماتيكى

\begin{tabular}{|c|c|c|c|c|}
\hline $\boldsymbol{\mu}$ & $\mathbf{P}$ & ميانكين \#|نحراف معيار & زمان & فاكتورها \\
\hline \multirow{2}{*}{$\cdot / N$} & \multirow{2}{*}{$\% 1^{*}$} & 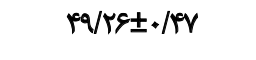 & قبل از خستكى & \multirow{2}{*}{ فلكشن } \\
\hline & & $9 / 19 \pm . / P A$ & بعد از خستكى & \\
\hline \multirow{2}{*}{$+/ n$} & \multirow{2}{*}{.$+.1^{*}$} & $|V| \cdot \Delta \pm \cdot / N$ & قبل از خستكى & \multirow{2}{*}{ و والكوس } \\
\hline & & $W N \Delta \Delta \pm+/ Y$ & بعد از خستكى & \\
\hline \multirow{2}{*}{$\cdot / A r$} & \multirow{2}{*}{$* . .1^{\circ}$} & NEA $=/ M$ & قبل ازخستكى & \multirow{2}{*}{ هرخش ساق ها } \\
\hline & & $|W / W \pm \cdot / Y|$ & بعد از خستكى & \\
\hline
\end{tabular}

مجله بيومكانيكووزش

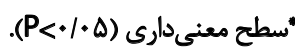

جدول r. نتايج اثرات مهارت بيشبينى بر فاكتورهاى كينماتيكى

\begin{tabular}{|c|c|c|c|c|}
\hline$\mu$ & $\mathbf{P}$ & ميانكين+|نحراف معيار & زمان & فاكتورها \\
\hline \multirow{2}{*}{ - /AF } & \multirow{2}{*}{$.1 . .1^{\circ}$} & $p q / r q \pm \cdot / p q$ & ييشينى شله & \multirow{2}{*}{ فلكشن } \\
\hline & & $P \& 1.8 \pm . / P V$ & ييشبينىنشله & \\
\hline \multirow{2}{*}{.$/ 49$} & \multirow{2}{*}{$.1 . .1^{\circ}$} & $I V / r \Psi \pm=/ N Y$ & ييشبينىشله & \multirow{2}{*}{ والكوس } \\
\hline & & WrVE./IF & ييشرينئشله & \\
\hline \multirow{2}{*}{. / A } & \multirow{2}{*}{$\%$} & NFE $\pm / 19$ & يشي يشينى & \multirow{2}{*}{ هرخش ساق يا } \\
\hline & & $11 / \Lambda \cdot \pm * / r$. & ييشرينيثشله & \\
\hline
\end{tabular}




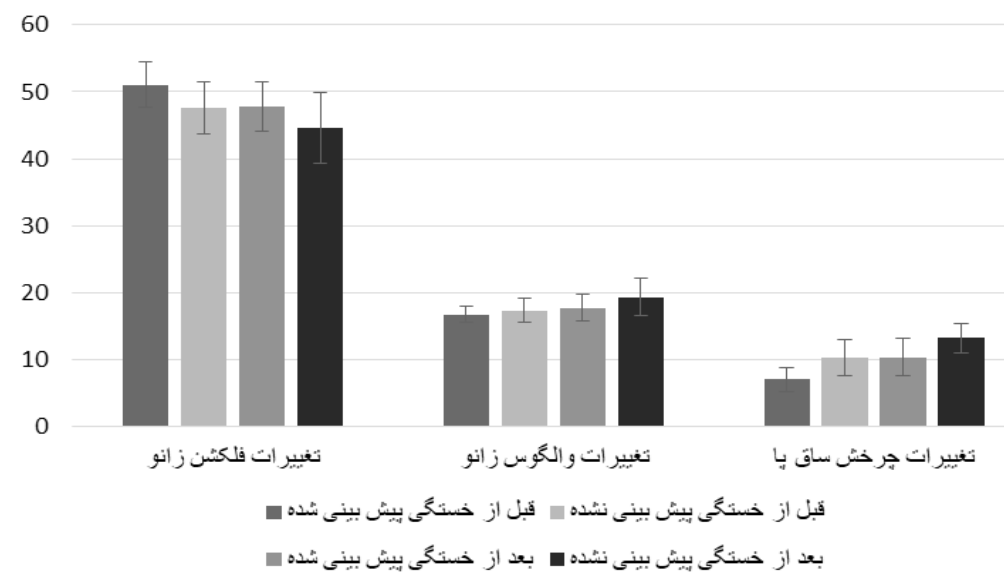

مجله بيومكانيك وزنث

راكنترل كند، با قرار دادن مفصل در موقعيت كاهش فلكشن زانو،

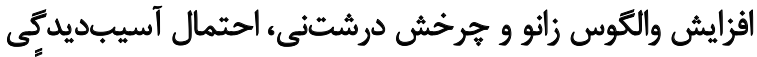

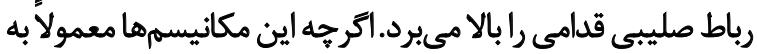
تنهايي مطالعه مي شوند، ليكن صدمه رباط صليبي قدي قدامي مي تواند

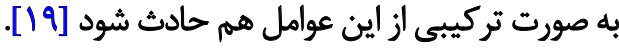

كو و همكاران كزارش كردند كه بعد از خستكي در مانور برش

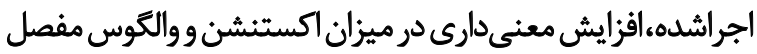

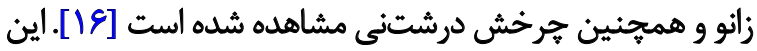

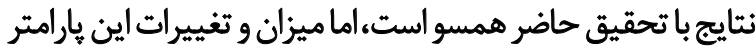

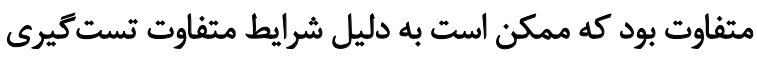

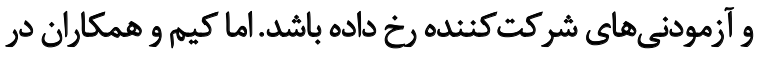

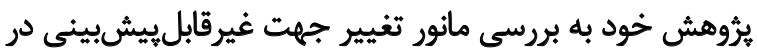

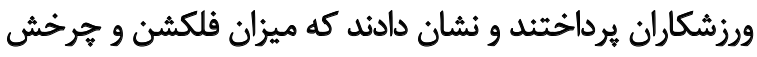

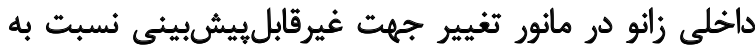

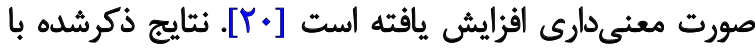
تحقيق حاضر همخوانى ندارد كه اين ميثتواند از اثرات متفاوت إنت

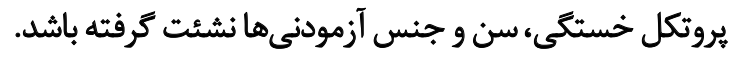
زاويه والكوس زانو هنگام فعاليتهاى ورزشى يكى از قوى رترين هيشبينى كنندهاى آسيب ليمامنت صليبى قدامى به شمار

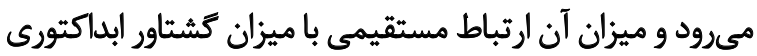

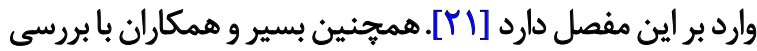

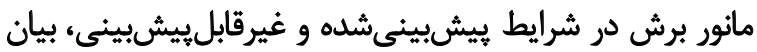

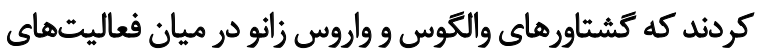

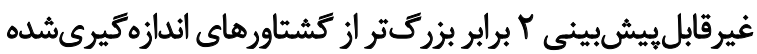

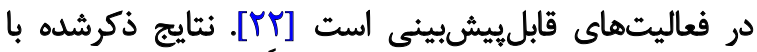

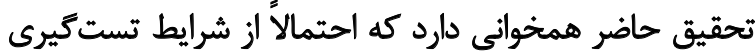

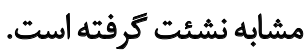

برخى ديكر از مطالعات، نيروى برشى تيبيا' را به عنوان

1. Anterior Shear Force
تصوير "ّ. ميزان تغييرات ميانكين هارامترهاى كينماتيكى در شرايط مختلف فاكتورهايي مانئ خستكي و فعاليتهاي غيرقابليشيشبيني

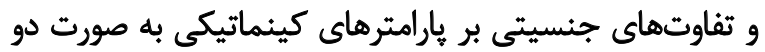

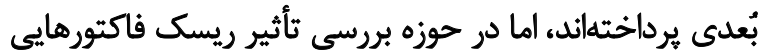

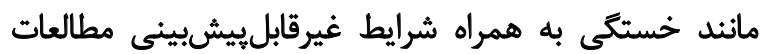

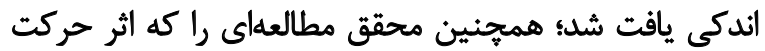

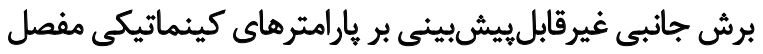

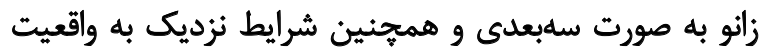

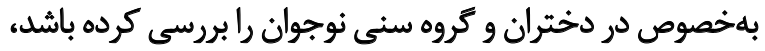

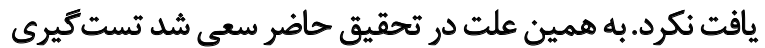

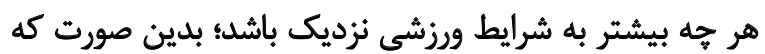

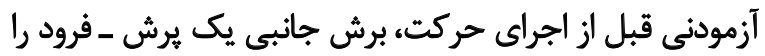

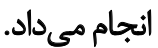

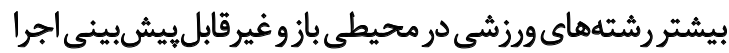

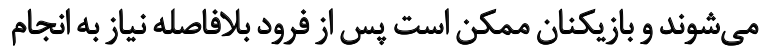

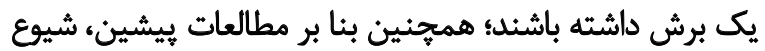

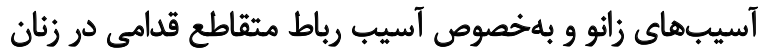

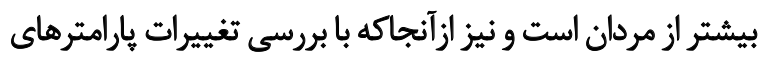

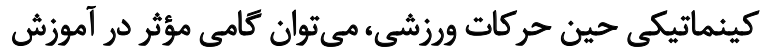

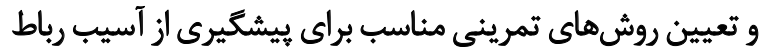

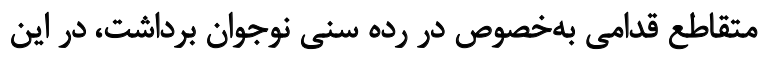

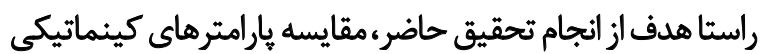

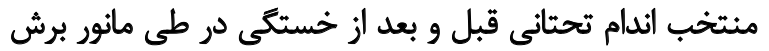

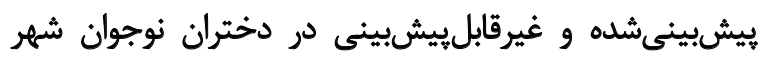

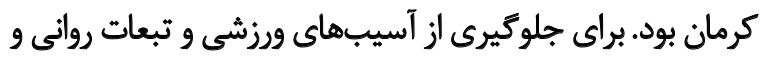

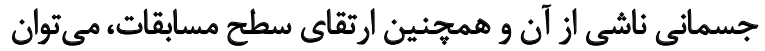

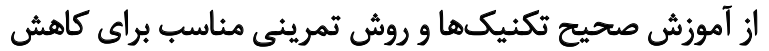

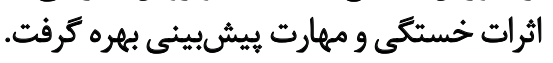

سازوكار آسيب رباط مثقاطع قدامى بيرو ايجاد فشار روى زانو

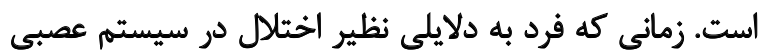

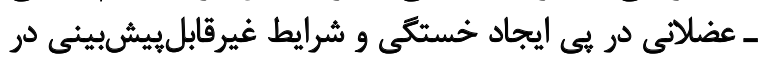
ورزش نمى تواندساختار اسكلتى-عضلانى وموقعيت صحيح مفصل 


\section{ماحظات اخلاقى - احق \\ يبيروى از اصول اخلاق يثوهش}

در اين مقاله كليه اصول اخلاقى در نظر كرفته شد.

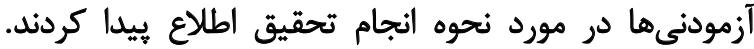

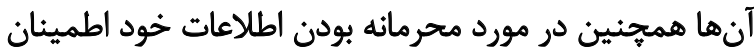

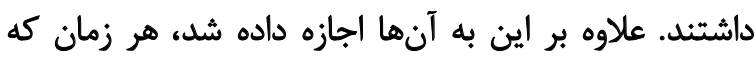

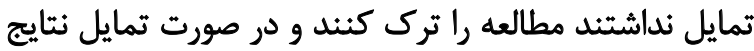

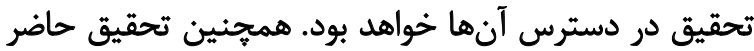

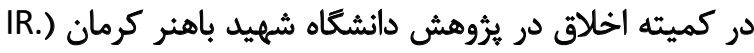

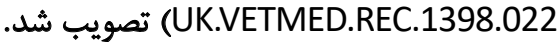

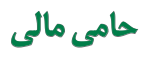

اين تحقيق هيج كمك مالى خاصى از سازمانهاى عمومى يا خصوصى دريافت نكرده است.

$$
\text { مشاركت نويسند مَان }
$$

مفهومسازى، روش شناسى ونظارت: تمامى نويسندكان؛ بررسى

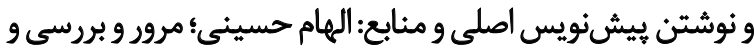
ويرايش: عبدالحميد دانشجو و منصور صاحب الزماني.

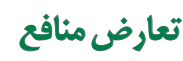

بنابر اظهار نويسندكان اين مقاله تعارض منافع ندارد.

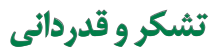

اين مقاله بر اساس بإيانثامه كارشناسى ارشد رشته

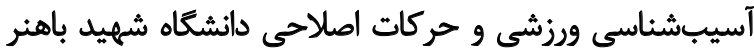

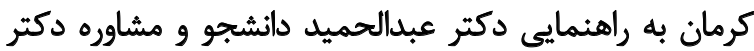

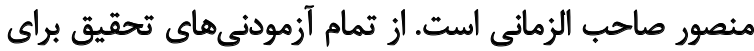

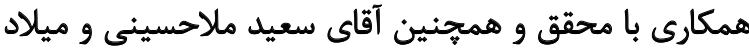

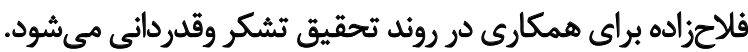

اصلي ترين عامل تعيينكنيده در ميزان بار وارده بر ليكامنت

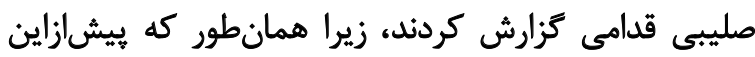

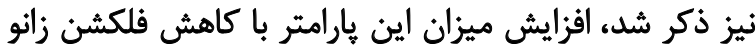

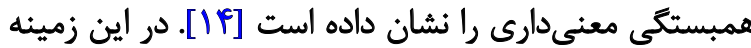

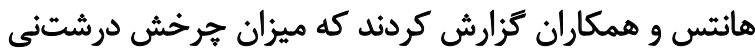

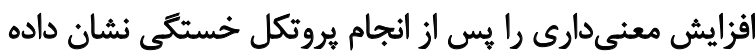

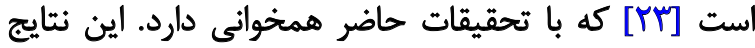

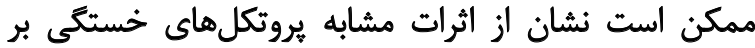

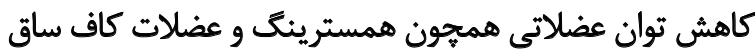

باشد كه وظيفه كنترل جرخش دران هرت همنى را برعهده دارند. ازآنجاكه مفصل زانو داراى سه درجه آزادى حركت است كه با

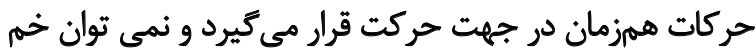

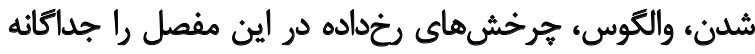

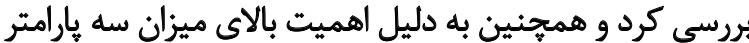

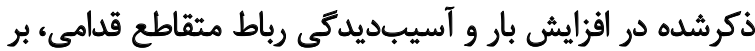

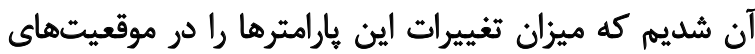

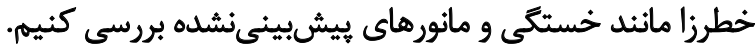

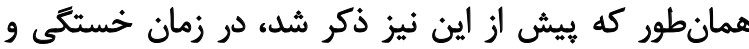

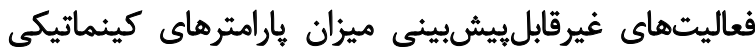

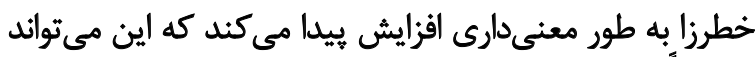

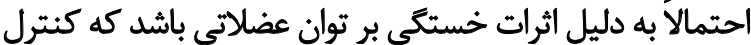

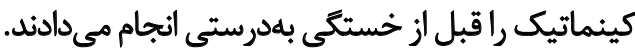

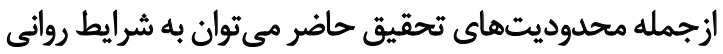

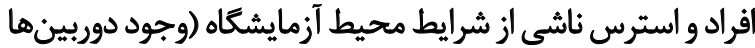

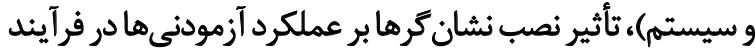

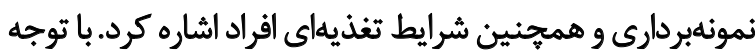

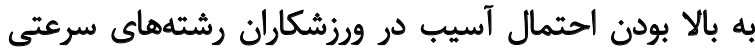

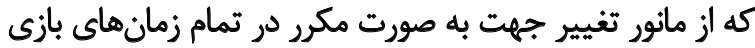

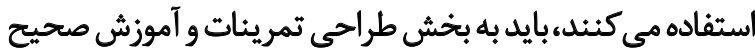

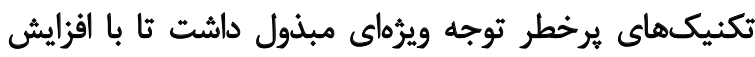
آكاهى بازيكنان از شرايط ايجاد آسيب جلوكيرى شئي شود.

\section{نتيجديرى نهايي}

نتايج بروهش حاضر نشان داد كه در زمان خستكى و مانورهاي

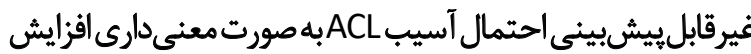

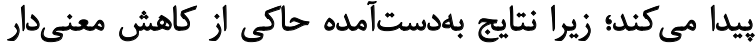

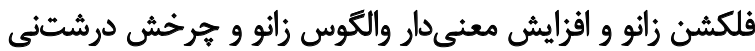

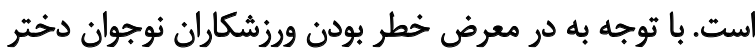

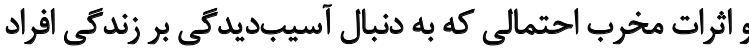

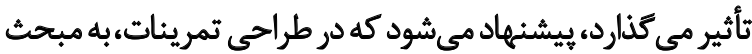

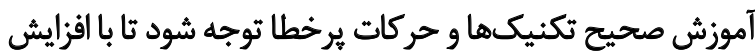

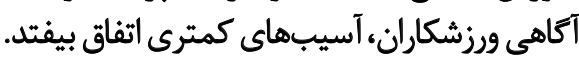




\section{References}

[1] Nuri L, Shadmehr A, Attarbashi Moghaddam B, Ghotbi N. Comparison of reaction time and anticipatory skill between female athletes and nonathletes. Mod Rehabil. 2012; 6(3):37-43.

[2] Arpante AK, Nguyen A-D, Baellow AL, Wright KE, Taylor JB, Ford KR. Effects of cutting direction on in-cleat toe dorsiflexion during unanticipated backpedal cutting in football players: 915 Board\# 311 May 27, 200 PM-330 PM. Med Sci Sports Exerci. 2015; 47(5S):253. [DOI:10.1249/01. mss.0000477116.36134.d3]

[3] Razi M, Sadeghi H, Takamejani EET, Shariatzade M. [Effect of lower limb muscle fatigue on knee joint control strategies during landing in young men (Persian)]. Sci J Rehabil Med. 2018; 7(2):1-10. [DOI:10.22037/ JRM.2018.110690.1459]

[4] Seyedi M, Rajabi R, Shirzad E, Zareei M. [Comparison of high-risk movement patterns of $A C L$ injury in male and female adolescent soccer players during cutting maneuver (Persian)]. J Sport Med Rev. 2016; 8(19):7794. http://www.magiran.com/p1657953

[5] Emami Hashemi SA, Rezvankhah Golsefidi N, Shirzad E, Mirkarimpour $\mathrm{SH}$. [Kinematic comparison of anterior cruciate ligament injury risk between men and women during cutting maneuver (Persian)]. Razi J Med Sci. 2015; 22(138):12-22. http://rjms.iums.ac.ir/article-1-4081-en.html

[6] Mok K-M, Bahr R, Krosshaug T. Reliability of lower limb biomechanics in two sport-specific sidestep cutting tasks. Sports Biomech. 2018, 17(2):157-67. [DOI:10.1080/14763141.2016.1260766] [PMID]

[7] Yom JP, Owens T, Arnett S, Beebe J, Son V. The effects of an unanticipated side-cut on lower extremity kinematics and ground reaction forces during a drop landing. Sports Biomech. 2019; 18(4):414-25. [DOI:10.108 0/14763141.2017.1409795] [PMID]

[8] Weir G, Stillman M, van Emmerik R, Wyatt $H$, Jewell C, Hamill J. Differences in kinetics, kinematics and muscle activation strategies in male and female team sport athletes during unanticipated sidestepping. I Sci Sport and Exerc. 2019; 1(2):159-67. [DOI:10.1007/s42978-019-0019-2]

[9] Buermann S, Gloppen E, Kriechbaum R, Potter D, Sheehan N. Influence of fatigue and anticipation on knee kinematics and kinetics during jump-cut maneuver. [Doctor of Physical Therapy Research Papers.]. Sophia: St. Catherine University; 2017

[10] Schneiders AG, Sullivan SJ, O'Malley KJ, Clarke SV, Knappstein SA, Taylor $\amalg$. A valid and reliable clinical determination of footedness. PM \& R 2010; 2(9):835-41. [DOI:10.1016/j.pmrj.2010.06.004] [PMID]

[11] Brown SR. The relationship between leg dominance and knee mechanics during the cutting maneuver. Indiana: Baal State University Muncie; 2012. https://www.semanticscholar.org/paper/ii-THE-RELATIONSHIP-BETWEEN-LEG-DOMINANCE-AND-KNEE-Wang/a26c3946d7d fa7947701ddce4c458d47ca015390

[12] Olsen O-E, Myklebust G, Engebretsen L, Bahr R. Injury mechanisms for anterior cruciate ligament injuries in team handball: $A$ systematic video analysis. Am J Sports Med. 2004; 32(4):1002-12. [DOI:10.1177/0363546503261724] [PMID]

[13] Winter DA. Biomechanics and motor control of human movement New Jersey: John Wiley \& Sons; 2009. [DOI:10.1002/9780470549148]

[14] Dahaji AA, Amirseyfaddini M, Nikooie R. Comparison of selected knee kinematic factors during single leg landing after six weeks of multi-angled isometric exercises on knee joint flexor and extensor muscle groups. Sci J Rehabil Med. 2019; 8(2):182-90. [DOI:10.22037/ jrm.2019.111456.2006]
[15] Sigward S, Powers CM. The influence of experience on knee mechanics during side-step cutting in females. Clin Biomech. 2006; 21(7):740-7. [DOI:10.1016/j.clinbiomech.2006.03.003] [PMID]

[16] Qu X, Chen X, editors. Combined effects of lower limb muscle fatigue and decision making to the knee joint during cutting maneuvers based on two different position-sense-acuity groups. International Conference on Applied Human Factors and Ergonomics. New York City: Springer 2017. [DOI:10.1007/978-3-319-60822-8_13]

[17] Fredriksen P, Ingjer F, Nystad W, Thaulow E. Aerobic endurance testing of children and adolescents-a comparison of two treadmill-protocols. Scand J Med Sci Sports. 1998; 8(4):203-7. [DOI:10.1111/j.1600-0838.1998.tb00193.x] [PMID]

[18] Chen MJ, Fan X, Moe ST. Criterion-related validity of the Borg ratings of perceived exertion scale in healthy individuals: a meta-analysis. J Sports Sci. 2002; 20(11):873-99. [DOI:10.1080/026404102320761787] [PMID]

[19] Cortes N, Onate J, Morrison S. Differential effects of fatigue on movement variability. Gait Posture. 2014; 39(3):888-93. [DOI:10.1016/j.gaitpost.2013.11.020] [PMID] [PMCID]

[20] Kim JH, Lee K-K, Ahn KO, Kong SJ, Park SC, Lee YS. Evaluation of the interaction between contact force and decision making on lower extremity biomechanics during a side-cutting maneuver. Arch Orthop Trauma Surg. 2016; 136(6):821-8. [DOI:10.1007/s00402-016-2457-1] [PMID]

[21] McLean S, Walker K, van den Bogert AJ. Effect of gender on lower extremity kinematics during rapid direction changes: An integrated analysis of three sports movements. J Sci Med Sport. 2005; 8(4):411-22. [DOI:10.1016/S1440-2440(05)80056-8]

[22] Besier TF, Lloyd DG, Cochrane JL, Ackland TR. External loading of the knee joint during running and cutting maneuvers. Med Sci Sports Exerc. 2001; 33(7):1168-75. [DOI:10.1097/00005768-200107000-00014] [PMID]

[23] Hantes ME, Tsarouhas A, Giakas G, Spiropoulos G, Sideris V, Christel P, et al. Effect of fatigue on tibial rotation after single-and double-bundle anterior cruciate ligament reconstruction: A 3-dimensional kinematic and kinetic matched-group analysis. Am J Sports Med. 2012; 40(9):2045 51. [DOI:10.1177/0363546512454413] [PMID] 